\title{
Tecnura
}

\section{Determinación de la incertidumbre del método de análisis de aflatoxinas por HPLC en pasa de uva}

\section{Definition of the uncertainty of the aflatoxin analysis method by HPLC in raisins}

\author{
Nora Dolores Martínez , Ana María Rodríguez², Aída Rosa Gutiérrez³, \\ Marina Daniela Di Carlo ${ }^{4}$, Analía de los Ángeles Durán ${ }^{5}$
}

Fecha de recepción: 18 de diciembre de 2017

Fecha de aceptación: 24 de mayo de 2018

\begin{abstract}
Cómo citar: Martínez, N.D., Rodríguez., A.M., Gutiérrez., A.R., Di Carlo., M.D y Durán., A.Á. (2018). Determinación de la incertidumbre del método de análisis de aflatoxinas por HPLC en pasa de uva. Tecnura, 22(58), 25-36. DOI: https://doi.org/10.14483/22487638.12896
\end{abstract}

\section{Resumen}

Contexto: En este trabajo se presenta la determinación de las incertidumbres de los métodos de análisis de aflatoxinas B1, B2, G1 y G2 en pasa de uva por HPLC-FLD. La incertidumbre de un método es uno de los parámetros requeridos por la norma IRAM 301-ISO/IEC 17025:2005, de aplicación en la República Argentina y acreditada por el Organismo Argentino de Acreditación (OAA). Forma parte de la validación del método analítico. Es un requisito necesario para ampliar el alcance de acreditación del Laboratorio de Análisis de Productos Regionales de Ingeniería Química (LAPRIQ), perteneciente al Instituto de Ingeniería Química de la Universidad Nacional de San Juan.

Metodología: El procedimiento utilizado es el denominado bottom up, basado en una sucesión de etapas de cálculo que tienen en cuenta los errores provenientes de cada una de las operaciones analíticas descompuestas en actividades primarias. La etapa inicial consiste en la identificación de las fuentes de error que pueden afectar el resultado del análisis. Se tomó como base para la determinación de las fuentes de error, y su incidencia en la incertidumbre final del proceso, el procedimiento de espina de pescado de Ishikawa. Definidos los factores que suman al error en la medición, se realizaron todos los ensayos de calibración del HPLC, índice de recuperación y verificación del instrumental para la determinación de la incertidumbre estándar de los subcomponentes. Se calculó luego la incertidumbre combinada teniendo en cuenta los aportes individuales. Posteriormente se sumaron las incertidumbres combinadas, ponderadas según la importancia de su contribución a través del coeficiente de sensibilidad. Por último, se determinó la incertidumbre expandida usando un factor de cobertura $\mathrm{K}=2$, que implica un porcentaje de confianza del $95 \%$. Con

1 Ingeniera química, docente e investigadora. Directora del Laboratorio de Análisis de Productos Regionales de Ingeniería Química (LAPRIQ). Profesora titular, Facultad de Ingeniería, Universidad Nacional de San Juan. San Juan, Argentina. Contacto: noramart@unsj.edu.ar

2 Ingeniera química, magíster en Ciencias de Superficie. Docente e investigadora. Profesora titular, responsable sección Análisis y Ensayos del Laboratorio de Análisis de Productos Regionales de Ingeniería Química (LAPRIQ), Facultad de Ingeniería, Universidad Nacional de San Juan. San Juan, Argentina. Contacto: arodri@unsj.edu.ar

3 Ingeniera química, docente e investigadora. Jefe área técnica del Laboratorio de Análisis de Productos Regionales de Ingeniería Química (LAPRIQ). Profesora titular, Facultad de Ingeniería, Universidad Nacional de San Juan. San Juan, Argentina. Contacto: agutierrez@unsj.edu.ar

4 Ingeniera química, especialista en Docencia Universitaria. Docente e investigadora. Analista Laboratorio de Análisis de Productos Regionales de Ingeniería Química (LAPRIQ). Jefe de trabajos prácticos, Facultad de Ingeniería, Universidad Nacional de San Juan. San Juan, Argentina. Contacto: marinadicarlo@gmail.com

5 Ayudante adscripta a investigación. Analista del Laboratorio de Análisis de Productos Regionales de Ingeniería Química (LAPRIQ), Facultad de Ingeniería, Universidad Nacional de San Juan. San Juan, Argentina. Contacto: analiaduran-17@hotmail.com 
este factor se puede esperar que se incluya la mayor fracción de la distribución de los valores atribuidos al mesurando.

Resultados: Se detectaron seis fuentes de error, correspondientes a las mediciones de: masa de la muestra, volúmenes de extracción, elución y alícuota para clean up, detección en HPLC e índice de recuperación del método. Las incertidumbres expandidas calculadas de las cuatro aflatoxinas son $\mathrm{AFB} 1=0,7 \mathrm{mg} / \mathrm{kg}, \mathrm{AFB} 2=0,2 \mathrm{mg} / \mathrm{kg}$, AFG1 = 0,9 mg/ $\mathrm{kg}$ y $\mathrm{AFG} 2=0,3 \mathrm{mg} / \mathrm{kg}$.

Conclusiones: Los valores de las incertidumbres expandidas se informan en el certificado de análisis, junto con el resultado del ensayo por HPLC, como una de las pruebas de la validación del método.

Palabras clave: aflatoxinas, HPLC, incertidumbre combinada, incertidumbre estándar, incertidumbre expandida.

\section{Abstract}

Objective: This work presents the definition of uncertainties for the methods of analysis of aflatoxins B1, B2, G1 and G2 in raisins by HPLC-FLD. The uncertainty of a method is one of the parameters required by the norm IRAM 301-ISO/IEC 17025:2005Standard, applicable in the Argentine Republic and accredited by the Argentine Accreditation Organism (OAA), and forms part of the validation of analytical methods. In addition, it is a necessary requirement to expand the scope of accreditation of the Laboratory of Analysis of Regional Products of Chemical Engineering (LAPRIQ, by the name in Spanish), belonging to the Institute of Chemical Engineering of the National University of San Juan.
Methodology: The procedure used is called "Bottom up", based on a succession of calculation that take into account errors from each of the analytical operations decomposed in primary activities. For the initial stage, sources of error that can affect the result of the analysis were identified. The Ishikawa's fishbone procedure was used as a basis for determining the sources of error and their incidence in the final uncertainty of the process. Once the factors that add up to the error in the measurement were defined, all the HPLC calibration tests were performed. Second, the combined uncertainties were added, weighted according to the importance of their contribution through the sensitivity coefficient. Finally, the expanded uncertainty was determined using a coverage factor $\mathrm{K}=2$, which implies a confidence percentage of $95 \%$. With this factor we can expect to include the largest fraction of the distribution of the values attributed to the measurand.

Results: Six sources of error were detected and correspond, on the one hand, to the mass measurements of the sample, extraction volumes, elution, and aliquot for "clean up" and, on the other, HPLC detection and recovery rate of the method. The calculated uncertainties for the four aflatoxins are $\mathrm{AFB} 1=0.7 \mathrm{mg} / \mathrm{kg}, \mathrm{AFB} 2=0.2 \mathrm{mg} / \mathrm{kg}, \mathrm{AFG} 1=0.9$ $\mathrm{mg} / \mathrm{kg}$ y $\mathrm{AFG} 2=0.3 \mathrm{mg} / \mathrm{kg}$.

Conclusions: The values of the expanded uncertainties are reported in the certificate of analysis, along with the result of the HPLC test, as one of the tests of the validation of the method validation.

Keywords: aflatoxins, HPLC, combined uncertainty, standard uncertainty, expanded uncertainty.

\section{INTRODUCCIÓN}

\section{La acreditación}

El desarrollo económico de la provincia de San Juan, República Argentina, está muy ligado a la industria de la uva y sus derivados, siendo la principal exportadora de pasas y mosto concentrado del país de acuerdo con lo informado por el Instituto Nacional de Vitivinicultura (INV, 2011). Los alimentos mencionados contienen principios bioactivos que resultan muy beneficiosos para la salud humana (Arias, Montaño, Velasco y Martínez, 2018). Los países importadores han aplicado 
condiciones de calidad cada vez más estrictas para aceptar los productos mencionados, teniendo en cuenta la importancia del cuidado de la salud de la población. Esto hace necesario que el exportador cuente con certificados de análisis respaldados internacionalmente, para garantizar que el producto cumple con los niveles de calidad acordados.

Disponer de certificados de análisis con respaldo internacional implica que el laboratorio que realiza los análisis cuente con la acreditación de la norma ISO 17025, específica para laboratorios de ensayo y calibración, otorgada por un organismo autorizado por el gobierno de cada país. El Organismo Argentino de Acreditación (OAA) es el único ente autorizado para acreditar la norma IRAM 301-ISO/IEC 17025, establecida por el Instituto de Racionalización de Materiales (IRAM, 2005).

El Instituto de Ingeniería Química de la Universidad Nacional de San Juan dispone del Laboratorio de Análisis de Productos Regionales de Ingeniería Química (LAPRIQ), acreditado por norma IRAM 301-ISO/IEC 17025 desde julio de 2013, con alcance en las técnicas de determinación de ocratoxina A para matriz pasa de uva (Rodríguez, Gutiérrez, Strazza y Giménez, 2008a) y matriz vino/mosto (Rodríguez, Gutiérrez, Strazza y Giménez, 2008b).

Es intención del grupo de trabajo solicitar al OAA la ampliación del alcance de acreditación para incluir la determinación de aflatoxinas en pasa de uva.

\section{Las aflatoxinas}

Uno de los requerimientos para el consumo de pasas de uva es la concentración de micotoxinas, sustancias consideradas el carcinógeno natural más potente conocido hasta el momento. Su producción está asociada al proceso de esporulación de hongos, a las condiciones ambientales y a la concentración de nutrientes en el medio (Bogantes-Ledezma, Bogantes-Ledezma y Bogantes-Ledezma, 2004).
Entre las micotoxinas vinculadas a la industria vitivinícola están las aflatoxinas, contaminantes orgánicos generados por hongos usualmente presentes en los viñedos de la región, especialmente en años donde las lluvias sobrepasan los valores normales promedio de precipitación (Martínez et al., 2015). Estas toxinas aparecen en la uva en fresco y, por ende, en pasas, vinos y mostos producidos a partir de ella. Hay al menos 99 países que tienen reglamentaciones para micotoxinas, cifra que corresponde al $87 \%$ de los habitantes del planeta (ONUAA, 2003).

En la vid naturalmente se encuentran hongos del tipo Aspergillus flavus, A. parasiticus y A. nominus. Estos mohos toxigénicos se desarrollan en gran variedad de sustratos, y se pueden producir aflatoxinas como metabolitos secundarios. El nombre de aflatoxina deriva de la abreviación taxonómica de Aspergillus y de la especie flavus. De las 18 variedades de aflatoxinas conocidas, la más tóxica es la aflatoxina B1 (AFB1). Las otras de interés en la pasa de uva son B2, G1 y G2. Tienen actividad inmonosupresiva, teratogénica, cancerígena y mutagénica; además, producen síndrome hepatotóxico y problemas renales (Gimeno y Martins, 2003).

Un comité mixto de la Organización de las Naciones Unidas para la Agricultura y la Alimentación y la Organización Mundial de la Salud (ONUAA, 2003) ha definido a las aflatoxinas como potentes carcinógenos humanos, aunque no existe aún información suficiente para establecer una cifra fija sobre grados de exposición tolerable. La mayoría de las leyes internacionales establecen un máximo de $4 \mathrm{mg} / \mathrm{kg}$ para aflatoxinas totales (la suma de B1, B2, G1, G2) en pasas de uva, no pudiéndose superar el límite de $2 \mathrm{mg} / \mathrm{kg}$ para la variedad B1, la más agresiva. El Código Alimentario Argentino no reglamenta el contenido de toxinas en pasas de uva (ANMAT, 2010). El Protocolo de Calidad de Pasas de Uva para la denominación Alimentos Argentinos mantiene los valores internacionales mencionados, como límites máximos de concentración y 
el resultado debe obtenerse por determinación en HPLC (SAGPyA, 2006).

En la figura 1 se muestra la representación de las moléculas de las cuatro aflatoxinas de interés. Pertenecen a la familia de las difurano-cumarinas, y son muy similares entre ellas, con diferencias en un enlace doble y en un anillo de 5 o 6 carbonos con inclusión de oxígeno. Químicamente, son estables y resistentes a la degradación bajo procedimientos de cocción normales, y es muy difícil eliminarlas una vez que se producen.

\section{El método de análisis}

El método de análisis de aflatoxinas para pasas por high performance liquid cromatography (HPLC) que se utiliza en el LAPRIQ sigue, en forma general, los lineamientos de la European Standard 14123:2008 (Aenor, 2008) con algunas modificaciones que responden al procedimiento sugerido por el proveedor de las columnas de inmunoafinidad (R-Biopharm, 2005a) y que requiere, por tanto, de una validación completa como método propio.
En la técnica hay tres pasos básicos: un proceso inicial de extracción con metanol, la posterior separación (clean up) en columna de inmunoafinidad (R-Biopharm, 2005b, 2006) y cuantificación por HPLC (Rodríguez y Gutiérrez, 2015). La columna de inmunoafinidad contiene anticuerpos monoclonales unidos a un soporte sólido que retiene las micotoxinas recuperadas luego con metanol. El HPLC tiene una columna cromatográfica donde las toxinas, según su afinidad con el soporte sólido, son retenidas con tiempos característicos diferentes para cada una. La toxina se somete posteriormente a la derivatización con bromo en una celda Kobracell@ (R-Biopharm, 2005c), para otorgarle características que permitan su detección en el detector por fluorescencia (FLD) y su cuantificación.

\section{La incertidumbre}

Una de las exigencias de la norma consiste en la validación del método analítico acreditado, lo que supone un importante diseño experimental para la
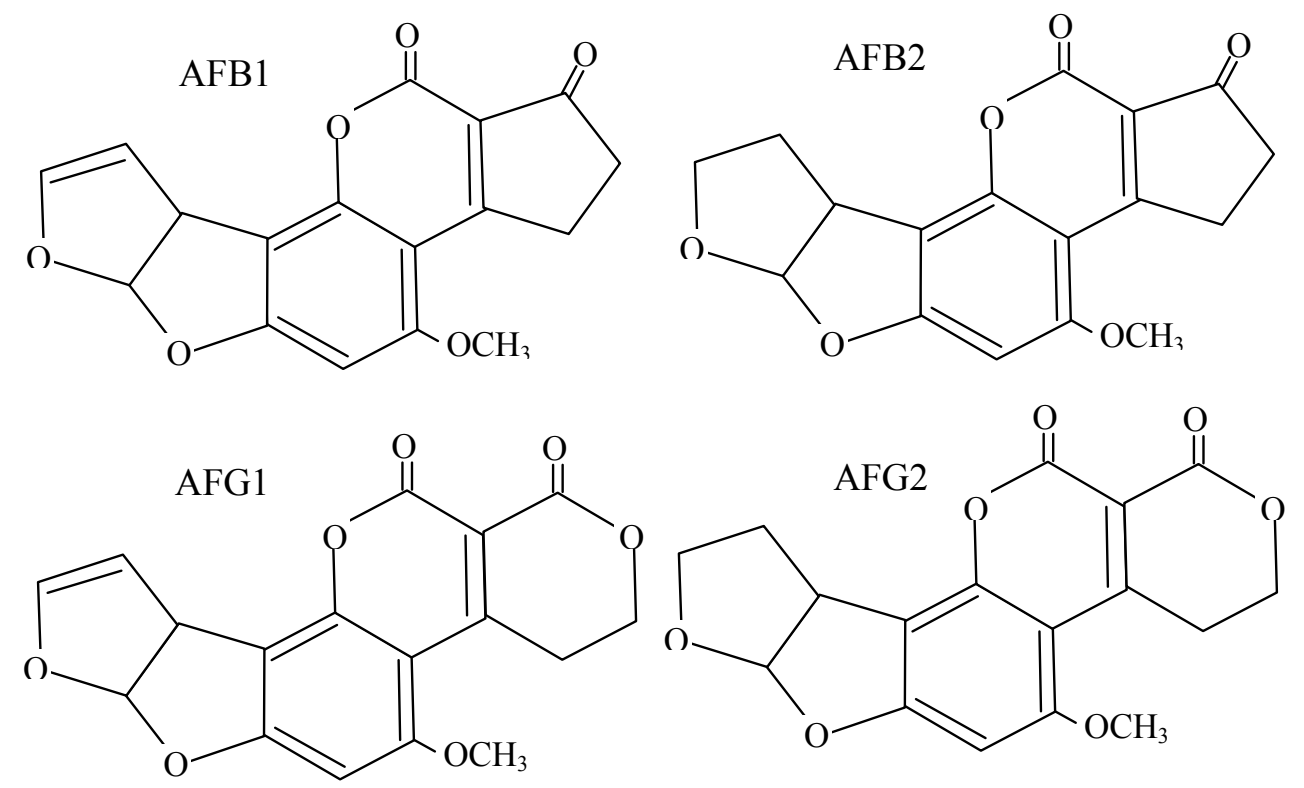

Figura 1. Estructura de las aflatoxinas

Fuente: elaboración propia. 
determinación estadística de los parámetros que fundamentan el aseguramiento de la calidad de la técnica, especialmente si se acredita como método propio. La validación es un procedimiento para demostrar científicamente por medio de estudios de laboratorio, que un método posee las características de desempeño adecuadas para cumplir con los requerimientos de las aplicaciones analíticas establecidas. La incertidumbre del proceso es uno de los requisitos para la validar un método.

Para determinar la incertidumbre se ha tomado como guía lo descrito en EURACHEM/CITAC Guide (2000). En líneas generales, el método de cálculo de la incertidumbre expandida de un ensayo consta de cuatro etapas. La primera consiste en la identificación de las fuentes de error que pueden afectar el resultado del análisis (mediciones críticas). Se calculó, luego, la incertidumbre combinada de cada una de las fuentes identificadas, teniendo en cuenta los aportes de incertidumbres estándar individuales. Posteriormente se sumaron las incertidumbres combinadas, ponderadas según la importancia de su aporte, a través del coeficiente de sensibilidad. Por último, se determinó la incertidumbre expandida que depende del porcentaje de confianza establecido y en el cual se puede esperar que se incluya la mayor fracción de la distribución de los valores atribuidos al mesurando. En el certificado de análisis entregado al productor junto con el resultado obtenido, se informa el valor de la incertidumbre expandida.

\section{METODOLOGÍA}

Para la estimación de la incertidumbre de un proceso de análisis químico hay dos métodos generales denominados top-down y bottom-up (CAC/ GL 59, 2006). El primero de ellos está basado en el procesamiento de datos registrados a largo plazo, derivados de resultados de ensayos de aptitud, muestras de control del laboratorio, datos bibliográficos publicados, etc. El LAPRIQ no cuenta todavía con información de largo plazo suficiente como para utilizar este método. El procedimiento bottom up, utilizado en este trabajo, se basa en el estudio exhaustivo de los errores provenientes de cada una de las operaciones analíticas descompuestas en actividades primarias. Este método, en contraposición con el método Top down, exige mayor grado de detalle o alta resolución (Carmona Aparicio et al., 2016).

Determinar la concentración final de un analito en una matriz implica calcular una función, la cual depende de las variables de cálculo (por ejemplo, volumen de extracción, respuesta del equipo, etc.). Para el cálculo de la concentración para cualquier aflatoxina, de acuerdo con el protocolo del método (Rodríguez y Gutiérrez, 2015), se utiliza la ecuación (1). La definición de todos los términos de esta y su valor se presentan en la sección "Resultados". Un análisis de aflatoxina implica determinar el valor de $C_{H P L C^{\prime}}$ que es la respuesta del cromatógrafo. Todos los demás términos son constantes porque son mediciones establecidas con valor único.

Concentración en $\frac{\mu g}{\mathrm{~kg}}=C_{x}=C_{H P L C} \frac{V_{1} \cdot V_{2}}{V_{3} \cdot M} \cdot \frac{1}{R}$

Todas las fuentes posibles de error identificadas y definidas a partir de la ecuación (1) se representaron en el diagrama de Ishikawa, también Ilamado espina de pescado.

Para cada uno de los componentes $\left(v_{i}\right)$ que aportan a la incertidumbre del proceso, se determinó la desviación estándar según ecuación (2) en la que $u_{m i}$ es la incertidumbre individual de cada factor que interviene en una medición específica (linealidad, temperatura, sensibilidad, etc.), de acuerdo con la ley de propagación de incertidumbres.

$$
u_{i}=\sqrt{\sum u_{m i}^{2}}
$$

Se realizaron todos los ensayos necesarios para determinar las incertidumbres estándar de cada medición, ya sea análisis químicos o verificación de medidas de masa o volumen. Cabe aclarar que todos los equipos e instrumentos utilizados en el LAPRIQ están calibrados y verificados según lo 
establecido en sus respectivos procedimientos de uso, los cuales regulan el procedimiento de trabajo y su frecuencia. Las calibraciones son externas y solo se realizan en organismos acreditados bajo norma IRAM 301-ISO/IEC 17025 para laboratorios de calibración.

Para calcular la incertidumbre combinada, se utilizó la ecuación (3), en la que cada incertidumbre estándar se multiplicó por un coeficiente de sensibilidad $c_{i}$ para ponderar su aporte a la incertidumbre global.

$$
u_{c}=\sqrt{\sum_{i}\left(c_{i} \cdot u_{i}\right)^{2}}
$$

Los coeficientes de sensibilidad se determinaron con la ecuación (4), derivándose la función $C_{x}$ definida en la ecuación (1), respecto a la componente $\left(v_{i}\right)$ cuyo coeficiente se quiere calcular.

$$
c_{i}=\frac{d C_{x}}{d v_{i}}
$$

La incertidumbre del método, denominada incertidumbre expandida, es el intervalo dentro del cual se asegura que se encuentra el resultado de la medición del mensurando $C_{x}$ con un grado de confianza especificado. Este valor aparece en el certificado de análisis y se calcula como la incertidumbre combinada multiplicada por el factor de cobertura $K$ según la ecuación (5). Se tomó $K=2$ asegurándose un 0,9545 \% de confianza en la medición. Este valor es común en la determinación de incertidumbres en otros ámbitos tales como la evaluación del error en las pérdidas eléctricas (Valencia y Avendaño, 2015).

$$
U_{C x}=K \cdot u_{c}
$$

Finalmente se determinó la contribución de cada variable a la incertidumbre total del proceso a través del índice de contribución de cada una de ellas (ecuación (6)); así se establecieron los puntos críticos que pueden mejorarse para disminuir la incertidumbre del método.

$$
\operatorname{Ind} \%=\frac{\left(c_{i} \cdot u_{i}\right)}{\sum_{i}\left(c_{i} \cdot u_{i}\right)} \cdot 100
$$

\section{RESULTADOS}

En la tabla 1 se define cada uno de los términos

\begin{tabular}{|c|c|c|c|}
\hline Símbolo & \multicolumn{2}{|l|}{ Descripción } & Valor \\
\hline $\mathrm{M}$ & \multicolumn{2}{|l|}{ Cantidad de fruta analizada } & $42 \mathrm{~g}$ \\
\hline $\mathrm{V}_{1}$ & \multicolumn{2}{|c|}{ Volumen de elución $(1,5 \mathrm{ml}$ de metanol HPLC $+1,5 \mathrm{ml}$ de agua HPLC) } & $3 \mathrm{ml}$ \\
\hline$V_{2}$ & \multicolumn{2}{|c|}{ Volumen de extracción (200 ml de metanol p.a. + 50 ml de agua HPLC) } & $250 \mathrm{ml}$ \\
\hline $\mathrm{V}_{3}$ & \multicolumn{2}{|c|}{ Alícuota del volumen de elución analizado } & $10 \mathrm{ml}$ \\
\hline \multirow{4}{*}{$\mathrm{R}$} & \multirow{4}{*}{ Índice de recuperación } & AFB1 & 0,9350 \\
\hline & & AFB2 & 0,9529 \\
\hline & & AFG1 & 0,9320 \\
\hline & & AFG2 & 0,9630 \\
\hline \multirow{4}{*}{$\mathrm{C}_{\mathrm{HPLC}}$} & \multirow{4}{*}{$\begin{array}{l}\text { Concentración medida en el HPLC para el valor de máximo } \\
\text { coeficiente de variación. }\end{array}$} & AFB1 & $1,6.10^{-9} \mathrm{~g} / \mathrm{ml}$ \\
\hline & & AFB2 & $2.10^{-10} \mathrm{~g} / \mathrm{ml}$ \\
\hline & & AFG1 & $1,6.10^{-9} \mathrm{~g} / \mathrm{ml}$ \\
\hline & & AFG2 & $2.10^{-10} \mathrm{~g} / \mathrm{ml}$ \\
\hline
\end{tabular}
de la ecuación (1). A partir de dicha ecuación, se definieron los componentes $\left(v_{i}\right)$ identificados como

Tabla 1. Variables de la expresión de cálculo de la concentración final

Fuente: elaboración propia. 
aportes a la incertidumbre del proceso: $M, V_{1}, V_{2}$ $V_{3,} R$ y $C_{H P L C}$. En la misma tabla se informan, además, los valores de los componentes utilizados más adelante para el cálculo de los coeficientes de sensibilidad. En la figura 2 se presenta el diagrama espina de pescado definido para este proceso. Todas las medidas dimensionales, se presentan en $\mathrm{ml}$ y $\mathrm{g}$.

De este modo quedan definidas las incertidumbres estándar que aportan a la incertidumbre combinada. Ellas son: $u_{M^{\prime}} u_{V 1^{\prime}} u_{V 2^{\prime}} u_{V 3^{\prime}} u_{R^{\prime}}$ y $u_{H P L C}$. Las cuatro primeras son comunes a todas las aflatoxinas. En este trabajo no se detalla, por su extensión, el cálculo de los aportes a las incertidumbres estándar. Se presentan solamente las ecuaciones y el resultado.

Todos los aportes individuales de error que se mencionan están definidos en procedimientos del
LAPRIQ que conforman el Sistema de Gestión de la Calidad. Las pruebas realizadas y los correspondientes cálculos descritos en los procedimientos están debidamente registrados y guardados en el laboratorio.

Los resultados de las incertidumbres estándar obtenidas se presentan en la tabla 2 .

\section{Determinación de uM}

La balanza analítica utilizada es marca Mettler Toledo modelo AL204.

Su incertidumbre estándar (ecuación (7)), informada en gramos, se calcula como la suma de las incertidumbres aportadas por el patrón, la linealidad, la sensibilidad y el estudio de R y R.

$$
\begin{gathered}
u_{m}=\sqrt{u_{\text {sen }}^{2}+u_{\text {lin }}^{2}+u_{\text {RyR }}^{2}+u_{\text {pat }}^{2}} \\
u_{m}=\sqrt{\left(2,88675.10^{-5}\right)^{2}+\left(1,73205.10^{-4}\right)^{2}+\left(8,57321.10^{-5}\right)^{2}+\left(3.10^{-5}\right)^{2}}
\end{gathered}
$$

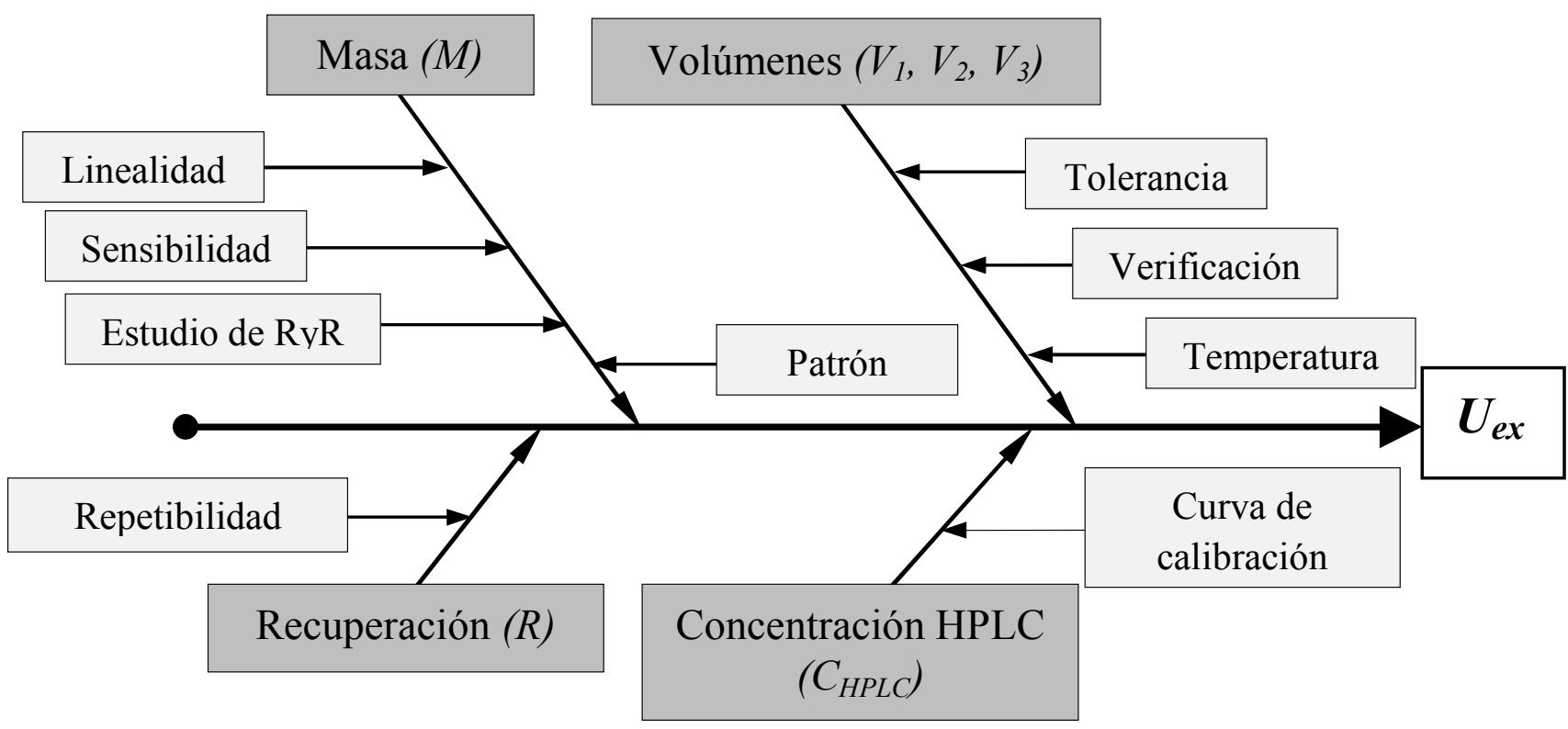

Figura 2. Diagrama de Ishikawa para el proceso de análisis

Fuente: elaboración propia. 


\section{Determinación de uV1}

El volumen de elución $V_{1}$ se mide con una micropipeta Transferpette de 1,0 ml. Se utiliza cuatro veces para tener la medición total de $3 \mathrm{ml}$ requerida, dos veces con metanol y dos veces con agua. La incertidumbre de cada medición está conformada por tres subcomponentes: tolerancia, variación en la temperatura (metanol y agua) y desviación por verificación, según la ecuación (8). Estos aportes se repiten en todas las mediciones de volumen con pipeta o micropipeta medidas en $\mathrm{ml}$.

$$
u_{V 1}=\sqrt{4 u_{\text {tot }}^{2}+2 u_{\text {Tmetanol }}^{2}+2 u_{\text {Tagua }}^{2}+4 u_{\text {ver }}^{2}}
$$

El aporte debido a la tolerancia se calculó de acuerdo con la ecuación (9).

$$
u_{\text {tol }}=\frac{\text { Tolerancia del fabricante }(\mathrm{ml})}{\sqrt{3}}
$$

La incertidumbre por variación de temperatura se calculó según la ecuación (10), donde a es el coeficiente de dilatación volumétrico correspondiente al fluido que se mide en $\mathrm{K}^{-1}$. Por razones de extensión no se detalla su cálculo.

$u_{\text {Tmetanol }}=\frac{\text { Variación de volumen }(\mathrm{ml})}{\sqrt{3}}=\frac{V_{\text {nominal }} \alpha \Delta T}{\sqrt{3}}$

Para la incertidumbre por verificación se a doptó para el cálculo la ecuación (11), método de evaluación tipo A (Delgado y Hernández, 2009).

$$
u_{\text {ver }}=\frac{s}{\sqrt{n}}
$$

Donde $s$ es la desviación estándar de las mediciones realizadas en la verificación del instrumental y $n$ es el número de mediciones.

$$
u_{V 1}=\sqrt{4\left(3,46410 \cdot 10^{-3}\right)^{2}+2\left(3,91010 \cdot 10^{-3}\right)^{2}+2\left(6,36528.10^{-4}\right)^{2}+4\left(5,48800 \cdot 10^{-5}\right)^{2}}
$$

\section{Determinación de uV2}

El volumen $V_{2}$, mezcla de metanol y agua, se mide con dos matraces calibrados de 200 y $50 \mathrm{ml}$ respectivamente. La incertidumbre de esta medida se calcula según la ecuación (12). A su vez, las incertidumbres individuales de la medida de metanol y agua se determinaron según las ecuaciones (13) y (14).

$$
\begin{gathered}
u_{V 2}=\sqrt{u_{\text {metanol }}+u_{\text {agua }}} \\
u_{\text {metanol }}=\sqrt{u_{\text {tol }}^{2}+u_{\text {Tmetanol }}^{2}+u_{\text {ver }}^{2}} \\
u_{\text {agua }}=\sqrt{u_{\text {tol }}^{2}+u_{\text {Tagua }}^{2}+u_{\text {ver }}^{2}}
\end{gathered}
$$

La incertidumbre por tolerancia del matraz se determinó mediante la ecuación (15):

$$
u_{\text {tol }}=\sqrt{\left(\frac{\text { Tolerancia }}{\sqrt{6}}\right)^{2}}
$$

De acuerdo con lo expuesto, la determinación de la incertidumbre correspondiente a la medición del volumen $V_{2}$ es:

$$
\begin{gathered}
u_{\text {metanol }}=\sqrt{(0,1)^{2}+(1,04269)^{2}+(0,06)^{2}} \\
u_{\text {agua }}=\sqrt{(0,0358547)^{2}+(0,0424352)^{2}+\left(9,80306 \cdot 10^{-3}\right)^{2}} \\
u_{V 2}=\sqrt{1,04920+5,64129 \cdot 10^{-2}}
\end{gathered}
$$

\section{Determinación de uV3}

$V_{3}$ es el volumen de alícuota que se toma luego del centrifugado para iniciar la etapa de clean up. Se miden $10 \mathrm{ml}$ de mezcla, medidos con pipeta calibrada. El cálculo se realizó de acuerdo con la ecuación (16).

$$
\begin{gathered}
u_{\text {metanol }}=\sqrt{u_{\text {tol }}^{2}+u_{\text {Tmezcla }}^{2}+u_{\text {ver }}^{2}} \\
u_{\text {agua }}=\sqrt{(0,011547)^{2}+(0,029705)^{2}+\left(3,65813 \cdot 10^{-4}\right)^{2}}
\end{gathered}
$$




\section{Determinación de uR}

Para el cálculo de la incertidumbre de la exactitud del método se utilizó la ecuación (17), lo que requirió la realización de 24 determinaciones (n) con pasas fortificadas a concentraciones conocidas. Se tomó la desviación estándar de la reproducibilidad intralaboratorio (s) usando la evaluación de incertidumbre tipo A según la ecuación (11). La $u_{R}$ para cada toxina se muestra en la tabla 1 .

$$
u_{R}=\frac{s}{\sqrt{n}}
$$

\section{Determinación de uHPLC}

La curva de calibración correspondiente a cada toxina requirió de cinco ensayos con tres analistas diferentes en días diversos, usando soluciones calibrantes de $2 ; 4 ; 8$ y $10 \mathrm{mg} / \mathrm{l}$ de aflatoxinas totales. Se utilizó un patrón Trilogy® con concentración de $5.000 \mathrm{mg} / \mathrm{l}$ totales y relación AFB1:AFB2:AFG1:AFG2=4:1:4:1 respectivamente. A partir de las rectas de calibración obtenidas se determinó la incertidumbre individual en $\mathrm{g} / \mathrm{ml}$ de acuerdo con la ecuación (18), donde $S_{c}$ es la desviación estándar que depende de cada aflatoxina:

$$
u_{H P L C}=\frac{S_{c}}{\sqrt{3}}
$$

Definidas las incertidumbres individuales, se calcularon los coeficientes de sensibilidad correspondientes. En la tabla 3 se muestran las ecuaciones derivadas para su cálculo y los resultados obtenidos teniendo en cuenta los valores dados en la tabla 1.

Tabla 2. Incertidumbres estándar calculadas

\begin{tabular}{clc}
\hline$u_{M}=1,9769498 \cdot 10^{-4} \mathrm{~g}$ & $u_{R B 1}=2,4924023 \cdot 10^{-2}$ & $u_{H P L C B 1}=1,87583 \cdot 10^{-10}$ \\
\hline$u_{V 1}=8,9106802.10^{-3} \mathrm{ml}$ & $u_{R B 2}=2,0295416.10^{-2}$ & $u_{H P L C B 2}=4,0683473.10^{-11}$ \\
\hline$u_{V 2}=1,1056087775 \mathrm{ml}$ & $u_{R G 1}=2,9487976.10^{-2}$ & $u_{H P L C G 1}=2,402630.10^{-10}$ \\
\hline$u_{V 3}=0,032088134 \mathrm{ml}$ & $u_{R C 2}=2,908972.10^{-2}$ & $u_{H P L C G 2}=1,1351565.10^{-10}$ \\
\hline
\end{tabular}

Fuente: elaboración propia.

\begin{tabular}{|c|c|c|c|}
\hline Variable & Ecuación correspondiente & & \\
\hline \multirow{3}{*}{ M } & & AFB1 & $7,2756.10^{-11}$ \\
\hline & $c_{M}=\frac{\partial C_{x}}{\partial M}=\frac{C_{H P L C} \cdot V_{1} \cdot V_{2}}{U} \cdot \frac{1}{D}$ & AFB2 & $8,9237 \cdot 10^{-12}$ \\
\hline & $c_{M}-\frac{\overline{\partial M}}{V_{3} \cdot M^{2}} \cdot \bar{R}$ & AFG1 & $7,2991.10^{-11}$ \\
\hline \multirow{3}{*}{$\mathrm{V}_{1}$} & & $\begin{array}{l}\text { AFG2 } \\
\text { AFB1 }\end{array}$ & $\frac{8,8301 \cdot 10^{-12}}{1.0186 \cdot 10^{-0 .}}$ \\
\hline & $=\frac{\partial C_{x}}{C_{H P L C} \cdot V_{2}}, \frac{1}{1}$ & AFB2 & $1,2493.10^{-11}$ \\
\hline & $c_{V 1}=\overline{\partial V_{1}}=\overline{V_{3} \cdot M} \cdot \bar{R}$ & AFG1 & $1,0219.10^{-0 .}$ \\
\hline \multirow{4}{*}{$V_{2}$} & & AFG2 & $1,2362.10^{-1 C}$ \\
\hline & $\partial C_{x}-C_{H P L C} \cdot V_{1} 1$ & $\begin{array}{l}\text { AFB1 } \\
\text { AFB2 }\end{array}$ & $\frac{1,2223 \cdot 10^{-11}}{1.4992 \cdot 10^{-12}}$ \\
\hline & $\overline{V_{3} \cdot M} \cdot \bar{R}$ & AFG1 & $1,2262.10^{-11}$ \\
\hline & & AFG2 & $1,4835.10^{-12}$ \\
\hline \multirow{2}{*}{$V_{3}$} & $\partial C_{x} \quad C_{H P L C} \cdot V_{1} \cdot V_{2} 1$ & $\begin{array}{l}\text { AFB1 } \\
\text { AFB2 }\end{array}$ & $\frac{3,0558.10^{-16}}{3,7480.10^{-11}}$ \\
\hline & $c_{V 3}=\overline{\partial V_{3}}$ & AFG1 & $3,0656.10^{-11}$ \\
\hline \multirow{3}{*}{$\mathrm{R}$} & & $\begin{array}{l}\text { AFG2 } \\
\text { AFB1 }\end{array}$ & $\frac{3,7086.10^{-11}}{3,2682.10^{-05}}$ \\
\hline & $C_{D}=\frac{\partial C_{x}}{C_{H P L C} \cdot V_{1} \cdot V_{2}}=\frac{1}{1}$ & AFB2 & $3,9332.10^{-16}$ \\
\hline & $\overline{\partial R}=\overline{V_{3} \cdot M} \cdot \overline{R^{2}}$ & AFG1 & $3,2893.10^{-0 .}$ \\
\hline \multirow{3}{*}{$\mathrm{C}_{\text {HPLC }}$} & & AFB1 & $\begin{array}{l}3,8511.10 \\
1,90985\end{array}$ \\
\hline & $c_{c} \quad=\frac{\partial C_{x}}{=}=\underline{V_{1} \cdot V_{2}} \cdot \frac{1}{1}$ & AFB2 & 1,87398 \\
\hline & ${ }^{{ }^{C} C_{H P L C}}-\overline{\partial C_{H P L C}}-\overline{V_{3} \cdot M} \cdot \bar{R}$ & $\begin{array}{l}\text { AFG1 } \\
\text { AFG2 }\end{array}$ & $\begin{array}{l}1,91600 \\
1,85432\end{array}$ \\
\hline
\end{tabular}

Tabla 3. Valor de los coeficientes de sensibilidad

Fuente: elaboración propia. 
La incertidumbre estándar combinada calculada con la ecuación (3) y la incertidumbre expandida $U_{C X}$ determinada con la ecuación (5) para cada toxina se presentan en la tabla 4.

En la tabla 5 se muestra el resultado del cálculo del índice porcentual de contribución de las

Tabla 4. Incertidumbres combinada y expandida incertidumbres a la incertidumbre total, y en la figura 3 se muestra el diagrama de barras de aportes individuales para las cuatro toxinas. Según estos resultados, el aporte principal a la incertidumbre combinada para todas las toxinas radica en la obtención de la curva de calibración en el HPLC.

\begin{tabular}{ccc}
\hline Toxina & Incertidumbre combinada & Incertidumbre expandida \\
\hline AFB1 & $u_{c B 1}=0,367643 \mu \mathrm{g} / \mathrm{kg}$ & $U_{C \times B 1}=0,7 \mu \mathrm{g} / \mathrm{kg}$ \\
\hline AFB2 & $u_{c B 2}=0,076692 \mu \mathrm{g} / \mathrm{kg}$ & $U_{C \times B 2}=0,2 \mu \mathrm{g} / \mathrm{kg}$ \\
\hline AFG1 & $u_{c G 1}=0,470838 \mu \mathrm{g} / \mathrm{kg}$ & $U_{C \times G 1}=0,9 \mu \mathrm{g} / \mathrm{kg}$ \\
\hline AFG2 & $u_{c G 2}=0,210805 \mu \mathrm{g} / \mathrm{kg}$ & $U_{C \times G 2}=0,4 \mu \mathrm{g} / \mathrm{kg}$ \\
\hline
\end{tabular}

Fuente: elaboración propia.

Tabla 5. Cálculo del índice porcentual de contribución a las incertidumbres

\begin{tabular}{ccccc}
\hline \multirow{2}{*}{ Variable } & \multicolumn{5}{c}{ Índice \% } \\
\cline { 2 - 5 } & AFB1 & AFB2 & AFG1 & AFG2 \\
\hline $\mathrm{C}_{\text {HPLC }}$ & 75,8820 & 86,4421 & 78,0439 & 93,2916 \\
\hline $\mathrm{R}$ & 17,2533 & 9,0508 & 16,4438 & 4,9651 \\
\hline $\mathrm{V}_{2}$ & 1,9224 & 1,2622 & 1,5437 & 0,4882 \\
\hline $\mathrm{V}_{3}$ & 2,8624 & 1,8793 & 2,2984 & 0,7269 \\
\hline $\mathrm{V}_{1}$ & 2,0769 & 1,3636 & 1,6677 & 0,5274 \\
\hline $\mathrm{M}$ & 0,0030 & 0,0020 & 0,0024 & 0,0008 \\
\hline
\end{tabular}

Fuente: elaboración propia.

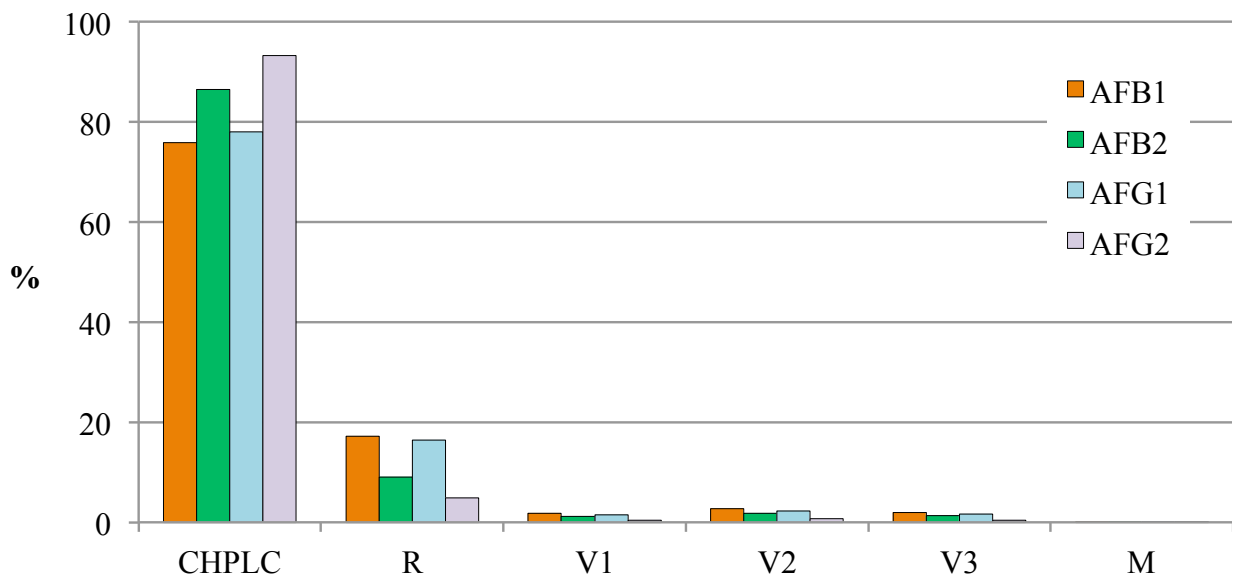

Figura 3. Representación de los aportes individuales a la incertidumbre combinada para todas las toxinas

Fuente: elaboración propia. 


\section{CONCLUSIONES}

En este trabajo se determinaron las incertidumbres expandidas de los procesos de determinación de aflatoxinas B1, B2, G1 y G2 en matriz pasa de uva. Los valores encontrados para cada una de ellas respectivamente son: $0,7 \mathrm{mg} / \mathrm{kg}, 0,2 \mathrm{mg} / \mathrm{kg}$, $0,9 \mathrm{mg} / \mathrm{kg}$ y $0,4 \mathrm{mg} / \mathrm{kg}$. Los resultados están de acuerdo con los esperados según otros métodos de determinación de toxinas que se realizan en el laboratorio. La determinación de las curvas de calibración en el HPLC es la actividad primaria que más aporta a la incertidumbre total, seguida por el índice de recuperación. De hecho, para simplificar el cálculo podrían despreciarse los aportes de las medidas de volumen y de masa, este último definitivamente insignificante, y considerar solo los dos de mayor contribución. Cabe aclarar que la determinación de las incertidumbres de la curva de calibración y del índice de recuperación, conllevan un arduo trabajo tanto experimental como estadístico, que no se ha descrito aquí por razones de extensión. Si bien no son tan laboriosas, las determinaciones de las demás incertidumbres estándar tampoco se han detallado en esta presentación por su longitud. Toda la información está disponible en el LAPRIQ.

El resultado de este trabajo aparece en el certificado de análisis que se entrega al productor, como prueba de la validación del método analítico.

\section{FINANCIAMIENTO}

Este trabajo se realizó en el marco del proyecto "Validación de los métodos de análisis para la cuantificación de aflatoxinas B1, B2, G1 y G2 en matriz pasa de uva y matriz vino/mosto por HPLC", financiado por el Consejo de Investigaciones Científicas, Técnicas y de Creación Artística (CICITCA) de la Universidad Nacional de San Juan.

\section{REFERENCIAS}

Administración Nacional de Medicamentos, Alimentos y Tecnología Médica (ANMAT) (1971). Código Alimentario Argentino. Decreto No. 2126/71 Reglamentario de la Ley Nacional No. 18.284. Buenos Aires, Argentina.

Arias L., D., Montaño D., L.N., Velasco S., M.A. y Martínez G., J. (2018). Alimentos funcionales: avances de aplicación en agroindustria. Revista Tecnura, 22(57), 55-68. DOI: https://doi. org/10.14483/22487638.12178

Asociación Española de Normalización y Certificación (Aenor) (2008). European Standard 14123:2008. Madrid, España.

Bogantes-Ledezma, P., Bogantes-Ledezma, D. y Bogantes-Ledezma, S. (2004). Aflatoxinas. Acta Médica Costarricense, 46(4), 174-178.

Carmona Aparicio, L.G., Rincón Pérez, M.A., Castillo Robles, A.M., Galvis Remolina, B.R., Sáenz Pulido, H.E., Manrique Forero, R.A. y Pachón Quinche, J.E. (2016). Conciliación de inventarios top-down y bottom-up de emisiones de fuentes móviles en Bogotá, Colombia. Revista Tecnura, 20(49), 59-74. DOI: 10.14483/udistrital.jour.tecnura.2016.3.a04

Delgado, G. y Hernández, N. (2009). Evaluación de la incertidumbre en la determinación de aflatoxina B1 en maní de exportación por HPLC-FD. Parte I. Universitas, 3(1), 5-15.

EURACHEM/CITAC Guide CG 4 QUAM:2000.1 (2000). Quantifying uncertainty in Analytical Measurement. 2a. ed. Reino Unido - Suiza.

Gimeno, A. y Martins, M.L. (2011). Micotoxinas y micotoxicosis en animales y humanos. 3a. ed. Miami, EE. UU.: Special Nutrients Inc.

Instituto Nacional de Vitivinicultura (INV) (2011). Las pasas de uva en el mundo y en Argentina. Mendoza, Argentina: Departamento de Estadística y Estudios de Mercado, Subgerencia de Estadística y Asuntos Técnicos Internacionales. 
Instituto de Racionalización de Materiales (IRAM) (2005). Norma IRAM 301-ISO/IEC 17025:2005. 4a. ed., Buenos Aires, Argentina: Editorial IRAM.

Martínez, N.D., Rodríguez, A.M., Venturini, R.B., Gutiérrez, A.R. y Granados, D.L. (2015). Abatement of Ochratoxin A from Contaminated Wine and Grape Juice by Activated Carbon Adsorption. Latin American Applied Research, 45, 33-38.

Organización de las Naciones Unidas para la Agricultura y la Alimentación (ONUAA) (2003). Reglamentos a nivel mundial para las micotoxinas en los alimentos. Recuperado de http://www.fao.org/docrep/ 007/ y5499s/y5499s07.htm.

Organización de las Naciones Unidas para la Agricultura y la Alimentación (ONUAA) (2006). CAC/ GL 59-2006: Directrices sobre la Estimación de la Incertidumbre de los Resultados. Recuperado de http://www.fao.org/input/download/standards/10692/ cxg059s.pdf.

R-Biopharm (2005a). Sultanas: Método de extracción para aflatoxinas. Buenos Aires, Argentina: R-Biopharm Rhöne Ltd. - R-Biopharm Latinoamérica.

R-Biopharm (2005b). Easy-Extract ${ }^{\circledast}$ Aflatoxin para la detección de aflatoxinas totales por HPLC. Buenos Aires, Argentina: R-Biopharm Rhöne Ltd. R-Biopharm Latinoamérica.

R-Biopharm (2005c). KOBRA CELL Celda electroquímica para la derivatización de aflatoxinas. Buenos Aires,
Argentina: R-Biopharm Rhöne Ltd. - R-Biopharm Latinoamérica.

R-Biopharm (2006). Aflatoxin clean up columns: Aplicación de columnas de clean up para la preparación de muestras previo al análisis de aflatoxinas por HPLC. Buenos Aires, Argentina: R-Biopharm Rhöne Ltd. - R-Biopharm Latinoamérica.

Rodríguez, A.M. y Gutiérrez, A.R. (2015). Análisis de aflatoxinas por HPLC en frutas desecadas. Documento interno PO-01-V4. LAPRIQ. San Juan, Argentina.

Rodríguez, A. M., Gutiérrez, A. R., Strazza, E. R. y Giménez, M. (2008a). Análisis de OTA por HPLC en Frutas Desecadas. Documento interno PO-03-V6. LAPRIQ. San Juan, Argentina.

Rodríguez, A. M., Gutiérrez, A. R., Strazza, E. R. y Giménez, M. (2008b). Análisis de OTA por HPLC en Vino y Mosto de Uva. Documento interno PO08-V3. LAPRIQ. San Juan, Argentina.

Secretaría de Agricultura, Ganadería, Pesca y Alimentos de la República Argentina (SAGPyA) (2006). Resolución No. 146 de 2006, Protocolo de Calidad para Pasas de Uva. Buenos Aires, Argentina.

Valencia, R. y Avendaño, C. (2015). Metodología para determinar la incertidumbre en la medición de pérdidas eléctricas para transformadores de distribución serie 15 kV. Revista Tecnura, 19(CITIE), 33-40. DOI: http://dx.doi.org/10.14483/udistrital. jour.tecnura.2015.ICE.a03.

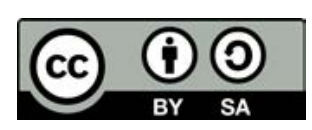

Tecnura • p-ISSN: 0123-921X • e-ISSN: 2248-7638 • Vol. 22 No. 58 • Octubre - Diciembre de $2018 \bullet$ pp. 25-36 